\title{
Emergency department occupancy is useful as a simple real-time measure of crowding
}

\author{
Robin Clouston ${ }^{1} \cdot$ Paul Atkinson $^{1} \cdot$ Donaldo D. Canales ${ }^{2} \cdot$ Jacqueline Fraser $^{3} \cdot$ Dylan Sohi $^{4} \cdot$ Scott Lee $^{5}$. \\ Michael Howlett ${ }^{1}$
}

Received: 15 May 2020 / Accepted: 5 February 2021 / Published online: 21 March 2021

(c) The Author(s), under exclusive licence to Canadian Association of Emergency Physicians (CAEP)/ Association Canadienne de Médecine d'Urgence (ACMU) 2021

\begin{abstract}
Introduction Emergency department (ED) crowding compromises patient outcomes. Existing crowding measures are complex and difficult to use in real-time. This study evaluated readily available single flow variables as crowding measures.

Methods Over 2 weeks in a tertiary Canadian ED, we recorded the following potential crowding measures during 168 consecutive two-hour study intervals: total ED patients (census), patients in beds, patients in waiting rooms, patients in treatment areas awaiting MD assessment; number of inpatients boarding, and ED occupancy. We also calculated four complex crowding scores-NEDOCS, EDWIN, ICMED, and a local modification of NEDOCS. We performed ROC analyses to assess the predictive validity of these measures against a reference standard of physician perception of crowding.

Results We gathered data for 144 (63.9\%) of 168 study intervals. ED census correlated strongly with crowding (AUC $=0.82$, 95\% CI 0.76-0.89), as did ED occupancy (AUC $=0.75,95 \%$ CI 0.66-0.83). Their performance was similar to NEDOCS $(\mathrm{AUC}=0.80)$ and to the local modification of NEDOCS $(\mathrm{AUC}=0.83)$.

Conclusion ED occupancy as a single measure has similar predictive accuracy to complex crowding scores and is easily generalizable to diverse emergency departments. Real-time tracking of this simple indicator could be used to prompt investigation and implementation of crowding interventions.
\end{abstract}

Keywords Crowding $\cdot$ Emergency department

\section{Résumé}

Introduction L'encombrement des services d'urgence (SU) compromet les résultats pour les patients. Les mesures d'encombrement existantes sont complexes et difficiles à utiliser en temps réel. Cette étude a évalué des variables de débit unique facilement disponibles comme mesures d'encombrement.

Les méthodes Pendant deux semaines dans un service d'urgence tertiaire canadien, nous avons enregistré les mesures d'encombrement potentiel suivantes au cours de 168 intervalles d'étude consécutifs de deux heures : nombre total de patients dans le service d'urgence (recensement), patients dans les lits, patients dans les salles d'attente, patients dans les zones

Robin Clouston

robin.clouston@dal.ca

1 Department of Emergency Medicine, Dalhousie University, Saint John Regional Hospital, 400 University Avenue,

Saint John, NB E2L 4L2, Canada

2 Research Services, Horizon Health Network, Saint John, NB, Canada

3 Department of Emergency Medicine, Horizon Health Network, Saint John, NB, Canada

4 Memorial University, St John's, NL, Canada

5 Dalhousie University, Halifax, NS, Canada 
de traitement en attente d'une évaluation médicale ; nombre de patients hospitalisés en internat et occupation du service d'urgence. Nous avons également calculé quatre scores de surpeuplement complexes : NEDOCS, EDWIN, ICMED et une modification locale de NEDOCS. Nous avons effectué des analyses ROC pour évaluer la validité prédictive de ces mesures par rapport à une norme de référence de perception du surpeuplement par les médecins.

Résultats Nous avons recueilli des données pour $144(63,9 \%)$ des 168 intervalles d'étude. Le recensement des urgences est fortement corrélé avec le surpeuplement (ASC $=0.82$, IC $95 \%=0.76-0.89$ ), tout comme l'occupation des urgences (ASC $=0.75$, IC $95 \%=0.66-0.83)$. Leur performance était similaire à celle des NEDOCS $(\mathrm{ASC}=0.80)$ et à la modification locale des NEDOCS (ASC $=0.83$ ).

Conclusion L'occupation des urgences en tant que mesure unique a une précision prédictive similaire aux scores complexes de surpeuplement et est facilement généralisable à divers services d'urgence. Le suivi en temps réel de ce simple indicateur pourrait être utilisé pour accélérer l'enquête et la mise en œuvre des interventions en cas de surpeuplement.

\section{Clinician's capsule}

What is known about the topic?

Validated measures of ED crowding are complex, resource-intensive and difficult to implement in real time.

\section{What did this study ask?}

Can a single measure predict emergency department crowding as accurately as validated complex crowding scores?

\section{What did this study find?}

ED occupancy is strongly predictive for crowding and performs equally well to the validated NEDOCS score.

\section{Why does this study matter to clinicians?}

$E D$ occupancy is an easily available indicator, generalizable across EDs, that may provide a reliable realtime estimation of crowding, prompting early investigation and intervention.

\section{Introduction}

Emergency department (ED) crowding is a worldwide problem with implications for patient care, system capacity and staff satisfaction $[1,2]$. No consensus exists on how to best measure ED crowding. At least eight formal crowding scores have been developed with varying validity and applicability [2] but most are complex, time-consuming, and difficult to use in real-time. Indicators of crowding should be simple, easily available in real-time, and maintain reliability and accuracy across many EDs [3]. We examined the predictive validity of six single measures, as well as four complex crowding scores [4-6], for identifying ED crowding at a tertiary Canadian hospital.

\section{Methods}

\section{Crowding predictors}

Over a 2-week period in July 2014, research assistants recorded flow measures during 168 consecutive 2-h intervals. Research assistants documented six single measures, including ED census (total patients), patients in beds, patients in the waiting room, patients in treatment areas awaiting physician assessment, number of inpatients boarding, and ED occupancy, defined as ED census divided by staffed ED beds ( 42 during the day, 24 overnight). Research assistants also compiled three multivariable crowding scores: EDWIN (ED Work Index), NEDOCS (National ED Overcrowding Score), and ICMED (International Crowding Measure in EDs) [4-6], as well as our local saturation calculator (SAT) based on NEDOCS. Crowding score descriptions are available in the Methods Supplement.

\section{Outcome}

Our reference standard for crowding was physician perception, assessed using a $10 \mathrm{~cm}$ visual analogue scale (VAS) ( $0=$ not crowded; $10=$ extreme crowding), by asking the in-charge physician the following question: How crowded do you think this ED is at the moment? [6] Physicians were unaware of other measures being concurrently tracked. The team lead nurse independently provided a VAS crowding assessment for each relevant interval, and we calculated intraclass correlation coefficient to assure interrater reliability [7].

\section{Analysis}

In keeping with previous research [4-6], we dichotomized physician VAS ratings to designate non-crowded (VAS 0-4.9) and crowded (VAS 5-10) periods. We correlated each of the crowding measures with physician impression, 
and performed receiver operating characteristic (ROC) curve analysis to examine predictive validity. We reported area under the curve (AUC) values ranging from 0 to 1 , with 0.50 indicating chance prediction, and values of $0.56,0.64$, and 0.71 reflecting small, medium and large effect sizes. Prior to reporting, we checked for collinearity and autocorrelation, finding that neither were threats to the analysis.

\section{Results}

Research assistants were available to capture data for 144 of 168 possible two-hour intervals. Physicians defined 92 of these $(64 \%)$ as crowded. Intraclass correlation coefficient for VAS crowding assessments was 0.6 indicating moderate inter-rater agreement [7]. Single variables demonstrated effect sizes comparable to formal crowding scores and to SAT (Table 1). ED census emerged as the best single variable $(\mathrm{AUC}=0.82)$ and was similar to NEDOCS $(\mathrm{AUC}=0.80)$ and SAT (AUC $=0.83$ ). Considering estimate precision (confidence ranges), ED occupancy (census/beds, AUC $=0.75$ ) performed similarly to ED census, NEDOCs and SAT, and had stronger predictive validity than EDWIN or ICMED. Number of patients occupying ED beds $(\mathrm{AUC}=0.76)$ and number in the waiting room $(\mathrm{AUC}=0.79)$ also performed well, but inpatient admissions $(\mathrm{AUC}=0.60)$ and patients waiting to be seen (TBS) $(\mathrm{AUC}=0.59)$ were not significant predictors of crowding.

Table 1 Predictive validity of single variables and crowding scores for crowding $(N=144)$

\begin{tabular}{lll}
\hline & AUC & 95\% CI \\
\hline Single variables & & \\
ED Census & .82 & {$[.76-.89]$} \\
\# patients in ED beds & .76 & {$[.68-.84]$} \\
\# patients in waiting room & .79 & {$[.72-.87]$} \\
\# inpatient admits & .60 & {$[.49-.70]$} \\
\# patients waiting TBS & .59 & {$[.49-.68]$} \\
Occupancy & .75 & {$[.66-.83]$} \\
Crowding scores & & \\
EDWIN & .67 & {$[.57-.77]$} \\
ICMED & .65 & {$[.56-.74]$} \\
NEDOCS & .80 & {$[.72-.88]$} \\
SAT & .83 & {$[.76-.90]$} \\
\hline
\end{tabular}

$A U C$ area under the curve, $C I$ confidence interval, $E D$ emergency department, TBS to be seen, EDWIN Emergency Department Work Index, ICMED International Crowding Measure in Emergency Departments, NEDOCS National Emergency Department Overcrowding Score, SAT local Saturation Calculator

\section{Discussion}

Rapid recognition of crowding facilitates timely process intervention, improved efficiency, expedited patient access, and reduced adverse events. This study showed a significant correlation between ED occupancy, NEDOCS and physician perception of crowding. We found that single easily calculated measures-ED census, number of patients in ED beds, patients in the waiting room, and ED occupancy-performed comparably to the best complex crowding scores. Of these, ED occupancy, that is, the ratio of ED census to bed capacity, is the most generalizable to diverse EDs.

ED crowding is a serious problem, but how to measure it remains unclear. A recent survey of Australian and New Zealand ED directors [8] found that the biggest challenge in crowding measurement is the choice of metric. Waxman and colleagues suggested ED census is a useful measure by showing it is associated with the number of patients who left without being seen and ambulance diversion rates [9]. McCarthy and colleagues found that the composite of occupancy, left without being seen and ambulance diversion is a moderately accurate crowding measure; however, left without being seen and ambulance diversion are relatively infrequent and not useful as real-time indicators [10]. Complex crowding scores like those studied here can be electronically calculated in some systems, but are often onerous in the calculation, and ultimately do not seem to perform better.

One of the most critical factors influencing crowding in the ED is hospital access block. Innes and colleagues [1] proposed the concept of facility access gap to emphasize the need for system wide co-operation: accountability for the access block is shared throughout the system and managers must create marginal efficiencies to reduce the access gap. The causes of ED crowding are dynamic and may related to combinations of inflow, throughput and outflow, however, the access gap process requires timely indicators in order to improve access. ED Occupancy is a simple, readily available measure of overall crowding, and may provide this early indicator.

After identifying a crowding situation based on ED occupancy, it is helpful to ascertain the immediate contributors, because solutions and mitigating strategies differ based on whether crowding is caused by inflow, throughput or outflow factors, or some combination. Therefore, facilities should also track more specific measures that highlight these three causes of ED crowding: inflow ( $\mathrm{N}$ patients in the waiting room, ambulance offload time), throughput (wait time to see MD, time seen by MD to disposition time) and output ( $\mathrm{N}$ inpatient boarders, $\mathrm{ED}$ length of stay for boarders). Hospital systems could therefore 
first identify the existence of crowding in real time using Occupancy, then in a second stage examine the factors contributing to the local crowding circumstance and rapidly implement interventions like those suggested by Innes et al. [1].

\section{Limitations}

There is no gold standard measurement of ED crowding so, like multiple previous studies, we used ED physician perception [4-6]. Physician perception is pragmatic and reflects observations on time, occupancy and workload, although we found it had only moderate interobserver reliability [8]. In addition, because the same nurse and physician provided several VAS crowding estimates during each shift, these estimates were not independent and our reliability estimates may be artificially inflated. Future research should investigate alternatives to this subjective gold standard. Second, single variables and crowding scores are not mutually exclusive and have a high degree of conceptual overlap. Third, while physicians did not participate in the calculation of complex crowding scores, nursing team leaders were not blinded to the local SAT calculator; therefore, nurse VAS crowding evaluations may have been influenced by this NEDOCs-derived tool. Fourth, while crowding spans a continuum from absent to severe, we artificially downgraded VAS ordinal values to binary categories. Lastly, the study was performed over a 2-week interval at a single site. Our findings may not generalize to other sites and seasons; therefore external validation in different environments is required.

\section{Conclusion}

In this setting, single readily available flow measures performed as well as complex crowding scores. For reasons of generalizability, we suggest that ED occupancy is a valuable indicator to facilitate real-time crowding recognition, to drive rapid implementation of operational solutions, and for tracking and evaluation of crowding strategies over time.

Supplementary Information The online version contains supplementary material available at https://doi.org/10.1007/s43678-021-00098-8.

Acknowledgements The authors would like to acknowledge the support provided by Horizon Research Services including Denise
Leblanc-Duchin for assistance in study design and Joshua Murray and George Stoica for assistance with data analysis. Additionally, the authors acknowledge Dr. Grant Innes for his feedback and suggestions to enhance the clarity of the manuscript.

Financial support This research received no specific grant from any funding agency, commercial or not-for-profit sectors.

\section{Compliance with ethical standards}

Conflict of Interest None.

\section{References}

1. Innes GD, Sivilotti ML, Ovens H, McLelland K, Dukelow A, Kwok E, et al. Emergency overcrowding and access block: a smaller problem than we think. CJEM. 2019;21(2):177-85. https://doi.org/10.1017/cem.2018.446.

2. Boyle A, Beniuk K, Higginson I, Atkinson P. Emergency department crowding: time for interventions and policy evaluations. Emerg Med Int. 2012. https://doi.org/10.1155/2012/838610.

3. Stang AS, Crotts J, Johnson DW, Hartling L, Guttmann A. Crowding measures associated with the quality of emergency department care: a systematic review. Acad Emerg Med. 2015;22(6):643-56. https://doi.org/10.1111/acem.12682.

4. Bernstein SL, Verghese V, Leung W, Lunney AT, Perez I. Development and validation of a new index to measure emergency department crowding. Acad Emerg Med. 2003;10(9):938-42. https://doi.org/10.1197/S1069-6563(03)00311-7.

5. Weiss SJ, Derlet R, Arndahl J, Ernst AA, Richards J, FernándezFrankelton M, et al. Estimating the degree of emergency department overcrowding in academic medical centers: results of the National ED Overcrowding Study (NEDOCS). Acad Emerg Med. 2004;11(1):38-50. doi:https://doi.org/10.1197/S1069-6563(03) 00583-9.

6. Boyle A, Atkinson P, Verdejo CB, Chan E, Clouston R, Gilligan $\mathrm{P}$, et al. Validation of the short form of the International Crowding Measure in Emergency Departments: an international study. Eur J Emerg Med. 2019;26(6):405-11. https://doi.org/10.1097/MEJ00 00000000000579.

7. Nunnally JC, Bernstein IH. Psychometric Theory. 3rd ed. New York: McGraw-Hill; 1994.

8. Jones P, Wells S, Ameratunga S. Towards a best measure of emergency department crowding: lessons from current Australasian practice. Emerg Med Australas. 2018;30(2):214-21.

9. Waxman DA, Husk G, Akhtar S, Krishnamurthy C. Hourly emergency department census: a simple measure of crowding. Ann Emerg Med. 2004;44(4):S19.

10. McCarthy ML, Aronsky D, Jones ID, Miner JR, Band RA, Baren $\mathrm{JM}$, et al. The emergency department occupancy rate: a simple measure of emergency department crowding? Ann Emerg Med. 2008;51(1):15-24. 